\title{
Improving prediction accuracy of tumor classification by reusing genes discarded during gene selection
} Jack Y Yang1, Guo-Zheng Li*2,3, Hao-Hua Meng², Mary Qu Yang ${ }^{4}$ and Youping Deng 5

\begin{abstract}
Address: ${ }^{1}$ Harvard Medical School, Harvard University, Cambridge, Massachusetts 02140-0888 USA, ${ }^{2}$ School of Computer Engineering \& Science, Shanghai University, Shanghai 200072, China, ${ }^{3}$ Institute of Systems Biology, Shanghai University, Shanghai 200072, China, ${ }^{4}$ National Human Genome Research Institute, National Institutes of Health, U.S. Department of Health and Human Services, Bethesda, MD 20892, USA and ${ }^{5}$ Department of Biological Sciences, University of Southern Mississippi, Hattiesburg, MS 39406. USA
\end{abstract}

Email: Jack Y Yang - jyang@bwh.harvard.edu; Guo-Zheng Li* - gzli@shu.edu.cn; Hao-Hua Meng - mhhtj@shu.edu.cn; Mary Qu Yang - yangma@mail.nih.gov; Youping Deng -youping.deng@usm.edu

* Corresponding author

from The 2007 International Conference on Bioinformatics \& Computational Biology (BIOCOMP'07) Las Vegas, NV, USA. 25-28 June 2007

Published: 20 March 2008

BMC Genomics 2008, 9(Suppl I):S3 doi:I0.I 186/I47|-2164-9-SI-S3

This article is available from: http://www.biomedcentral.com/I47I-2I64/9/SI/S3

(c) 2008 Yang et al.; licensee BioMed Central Ltd.

This is an open access article distributed under the terms of the Creative Commons Attribution License (http://creativecommons.org/licenses/by/2.0), which permits unrestricted use, distribution, and reproduction in any medium, provided the original work is properly cited.

\begin{abstract}
Background: Since the high dimensionality of gene expression microarray data sets degrades the generalization performance of classifiers, feature selection, which selects relevant features and discards irrelevant and redundant features, has been widely used in the bioinformatics field. Multitask learning is a novel technique to improve prediction accuracy of tumor classification by using information contained in such discarded redundant features, but which features should be discarded or used as input or output remains an open issue.
\end{abstract}

Results: We demonstrate a framework for automatically selecting features to be input, output, and discarded by using a genetic algorithm, and propose two algorithms: GA-MTL (Genetic algorithm based multi-task learning) and e-GA-MTL (an enhanced version of GA-MTL). Experimental results demonstrate that this framework is effective at selecting features for multitask learning, and that GA-MTL and e-GA-MTL perform better than other heuristic methods.

Conclusions: Genetic algorithms are a powerful technique to select features for multi-task learning automatically; GA-MTL and e-GA-MTL are shown to to improve generalization performance of classifiers on microarray data sets.

\section{Background}

Tumor classification is performed on microarray data collected by DNA microarray experiments from tissue and cell samples [1-3]. The wealth of such data for different stages of the cell cycle aids in the exploration of gene inter- actions and in the discovery of gene functions. Moreover, genome-wide expression data from tumor tissues gives insight into the variation of gene expression across tumor types, thus providing clues for tumor classification of individual samples. The output of a microarray experi- 
ment is summarized as an $p \times n$ data matrix, where $p$ is the number of tissue or cell samples and $n$ is the number of genes. Here $n$ is always much larger than $p$, which degrades the generalization performance of most classification methods. To overcome this problem, feature selection methods are applied to reduce the dimensionality from $n$ to $k$ with $k<<n$.

Feature selection chooses a subset of the original features (genes) according to the classification performance; the optimal subset should contain relevant but non-redundant features. Feature selection can help to improve the generalization performance of classifiers, and to reduce learning time and the time required to classify out-of-sample data. There has been a great deal of work in machine learning and related areas to address this issue [4-7]. In most practical cases, relevant features are selected and kept as input, while irrelevant and redundant features are removed.

Although the removed features are redundant and weakly relevant, they contain useful information that can be used to improve prediction accuracy. Multi-Task Learning (MTL) is a method of using the redundant information by selecting features from the discarded feature set to add to the target $[8,9]$. Although MTL achieves only limited improvement, it is nevertheless useful for real world cases like medical problems [10] and multivariate calibration problems [11].

Previous studies of search methods for multi-task learning mainly used heuristic methods $[9,11]$, where the number of features selected for the input and/or target is somewhat arbitrary. When the search method is regarded as a combinational optimization problem, random search methods can be used. The genetic algorithm [12] is a simple and powerful method which has obtained satisfactory results for feature selection [13]. Motivated by this, we proposed the random method GA-MTL (Genetic Algorithm based Multi-Task Learning) [14], but GA-MTL did not consider irrelevant features in the data sets. Here we propose an enhanced version of GA-MTL (e-GA-MTL) which codes one feature with two binary bits. The e-GAMTL algorithm and others are applied to tumor classification on microarray data sets; it is found that e-GA-MTL outperforms all other algorithms considered.

\section{Results and discussion}

In order to demonstrate the benefits of multi-task learning methods, we have performed the following series of experiments using artificial neural networks (ANNs) as classifiers.

1. ALL is a baseline method; without any selection, all the genes are input to the ANN for classification.
2. GA-FS uses a genetic algorithm to select genes and input selected genes to the ANN.

3. H-MTL uses a heuristic embedded feature selection method to search features, where some of the selected features serve as input to the ANN and some of the features are added to the output.

4. GA-MTL uses a genetic algorithm to search features, where some of the selected features are input into ANN and some of the features are added to the output.

5. GA-MTL-IR uses an embedded algorithm to remove irrelevant features and then uses a genetic algorithm to search features, where some of the selected features serve as input to the ANN and some of the features are added to the output.

6. e-GA-MTL also uses a genetic algorithm to search features, and employs two bits to represent one feature; some features are considered as irrelevant and discarded, some of the selected features serve as input to the ANN, and some of the features are added to the output.

The most important parameter of an ANN is the number of nodes in hidden layer, $M$. To reduce the effect of this parameter we ran the experiments with both $M=2$ and $M$ $=10$.

While different data sets, including data sets with only the selected features, need different optimal parameters for different methods, we do not try to find the optimal parameters, because:

(1) It is infeasible to find the optimal parameters, because this is an NP-hard problem.

(2) We are not interested in obtaining the best performance of one special method on a given data set; instead, we are interested in demonstrating the effect of our proposed framework.

\section{Prediction performance}

The average BACC values are shown in Figures 1 and 2 for different values of the ANN parameters, where ALL means all the genes are used as input for classification without any gene selection. From Figures 1 and 2, we conclude that:

(1) On average and for all the data sets, the multi-task learning algorithms H-MTL, GA-MTL, GA-MTL-IR, and eGA-MTL perform better than the feature selection algorithms GA-FS and ALL. 


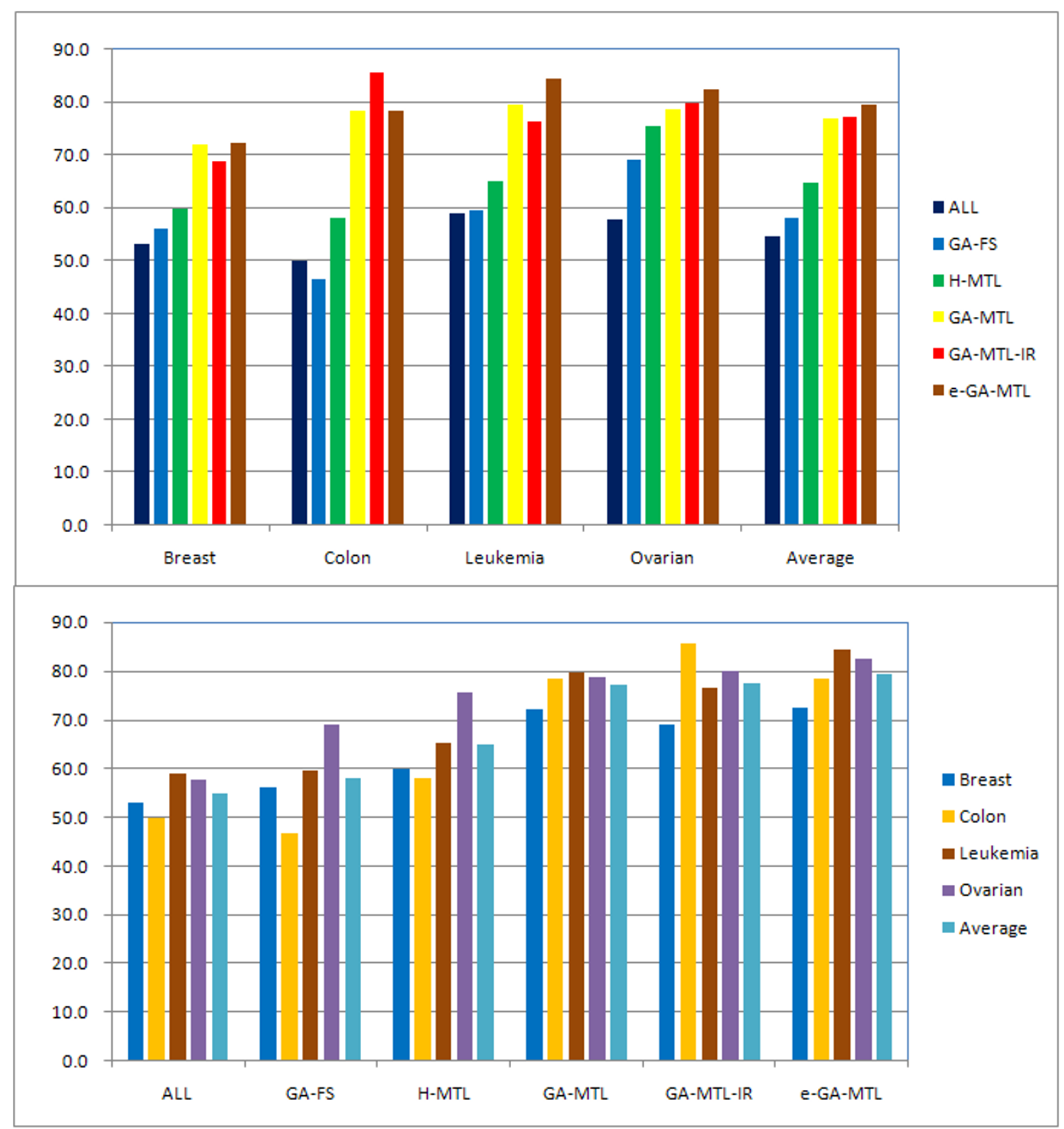

Figure I

Performance of multi-task learning algorithms for ANNs with $\mathbf{M}=\mathbf{2}$ hidden units. Both graphs show balanced accuracy (BACC) scores. Top: Results grouped by data set. Bottom: Results grouped by multi-task learning algorithm

(2) On average and for almost all the data sets, the genetic algorithm based multi-task learning algorithms GA-MTL, GA-MTL-IR and e-GA-MTL perform better than H-MTL, a heuristic algorithm. Only on the leukemia data set, for an ANN with $M=10$ hidden units, does H-MTL perform slightly better than GA-MTL and GA-MTL-IR.
(3) On average, e-GA-MTL performs the best among all the learning algorithms.

(4) Although GA-FS performs worse than the multi-task learning algorithms, it performs better than those without any gene selection. 


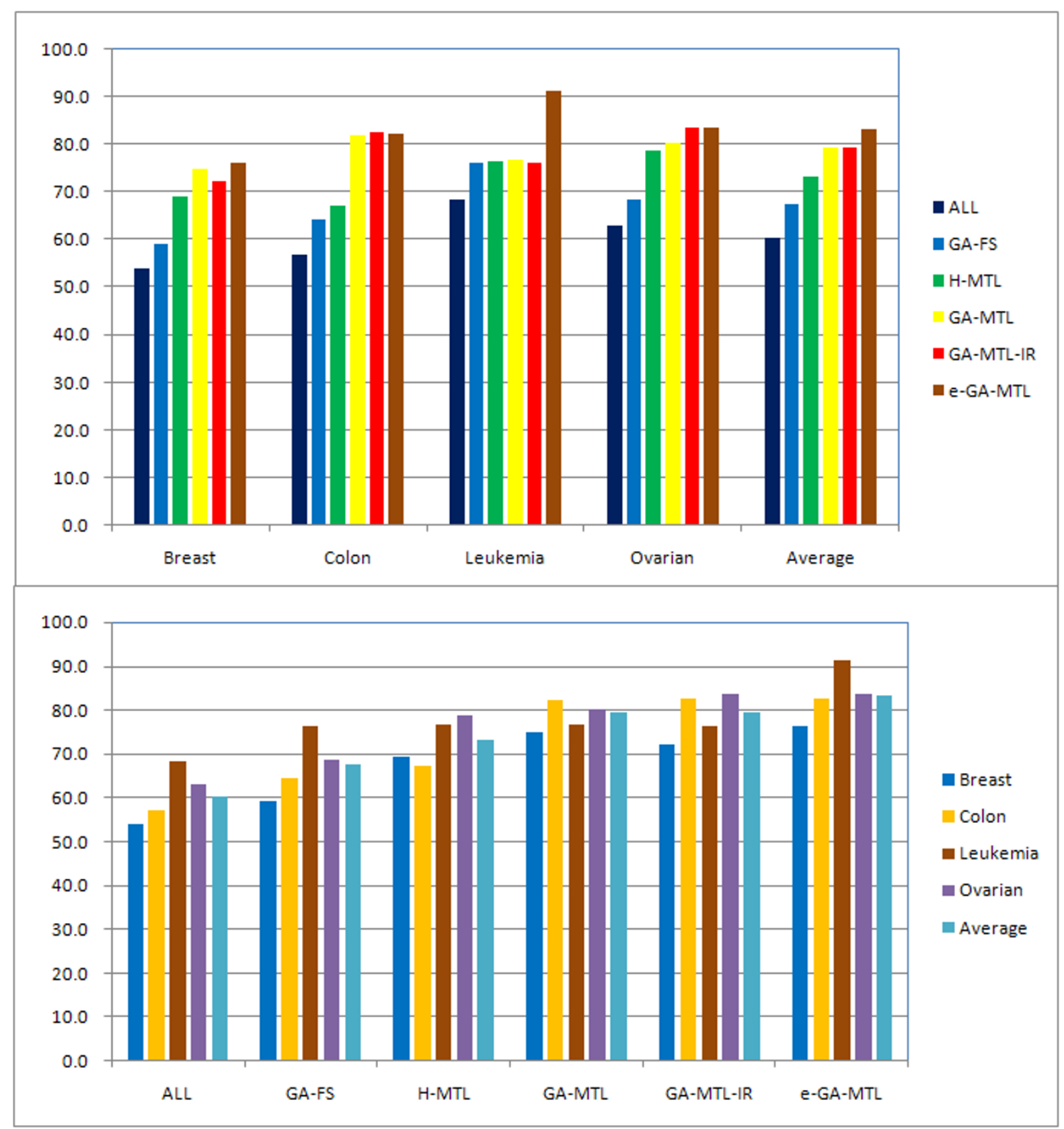

Figure 2

Performance of multi-task learning algorithms for ANNs with $\mathbf{M}=\mathbf{I} 0$ hidden units. Both graphs show balanced accuracy (BACC) scores. Top: Results grouped by data set. Bottom: Results grouped by multi-task learning algorithm.

Detailed statistical values of BACC, correction, sensitivity, specificity, and precision are also listed in Tables $1,2,3,4$ and 5, from which we conclude that:

(1) Although the results for ANNs with $M=10$ are better than those for $M=2$, we can draw similar conclusions for both series of results in terms of how the different methods compare.
(2) For all the measures, on average, multi-task learning algorithms including H-MTL, GA-MTL, GA-MTL-IR, and eGA-MTL perform better than GA-FS and ALL, and genetic algorithm based multi-task learning algorithms like GAMTL, GA-MTL-IR, and e-GA-MTL perform better than HMTL. 
Table I: Mean and standard deviation (in parentheses) of BACC scores (\%), calculated over $\mathbf{5 0}$ hold out runs.

\begin{tabular}{|c|c|c|c|c|c|c|}
\hline DATASET & ALL & GA-FS & H-MTL & GA-MTL & GA-MTL-IR & e-GA-MTL \\
\hline \multicolumn{7}{|c|}{$M=2$ for $\mathrm{ANN}$} \\
\hline Breast & $53.2(9.3)$ & $56.1(8.6)$ & $59.8(8.5)$ & $72.0(8.3)$ & $69.0(8.4)$ & $72.4(8.4)$ \\
\hline Colon & $50.0(8.8)$ & $46.7(8.5)$ & $58.1(7.9)$ & $78.6(8.4)$ & $85.7(8.1)$ & $78.6(7.8)$ \\
\hline Leukemia & $59.1(7.8)$ & $59.7(8.2)$ & $65.2(7.9)$ & 79.6(7.9) & $76.4(7.7)$ & $84.5(7.6)$ \\
\hline Ovarian & $57.8(6.8)$ & $69.1(6.8)$ & $75.6(3.8)$ & $78.9(7.2)$ & $79.9(7.1)$ & $82.4(7.1)$ \\
\hline Average & $54.8(8.2)$ & $58.1(8.0)$ & $64.8(7.8)$ & $77.1(7.9)$ & 77.4(7.8) & 79.5(7.7) \\
\hline \multicolumn{7}{|c|}{$M=10$ for $\mathrm{ANN}$} \\
\hline Breast & $54.2(9.3)$ & $59.3(8.9)$ & $69.2(9.2)$ & $74.9(8.8)$ & $72.3(9.3)$ & $76.2(8.7)$ \\
\hline Colon & $57.0(8.9)$ & $64.4(8.6)$ & $67.4(9.0)$ & $82.2(8.6)$ & $82.7(8.6)$ & $82.5(8.8)$ \\
\hline Leukemia & $68.4(8.1)$ & $76.2(7.7)$ & $76.6(8.0)$ & $76.8(7.5)$ & $76.4(7.1)$ & $91.4(7.2)$ \\
\hline Ovarian & $63.0(6.3)$ & $68.5(6.6)$ & $78.8(5.9)$ & $80.3(6.0)$ & $83.5(6.2)$ & $83.6(6.3)$ \\
\hline Average & $60.4(8.2)$ & $67.6(8.0)$ & $73.2(8.0)$ & $79.4(8.0)$ & $79.5(7.8)$ & $83.3(7.7)$ \\
\hline
\end{tabular}

(3) Both e-GA-MTL and GA-MTL-IR remove irrelevant genes; both obtain better results than the others for the specificity, precision and BACC measures, on average. But GA-MTL-IR performs worse than GA-MTL for other measures like sensitivity and correction.

(4) e-GA-MTL performs the best among all the learning algorithms on average, for all the measures. It greatly improves results for the BACC, sensitivity and specificity measures.

\section{The number of selected features}

We show the number of features selected by each algorithm in Tables 6 and 7, which also lists the number of discarded features, input features, and target features. For GA-FS, the features are selected as input or are discarded. For GA-MTL, the features are selected as input or are added to the target; no features are discarded. For H-MTL, GA-MTL-IR, and e-GA-MTL, the features are selected as input, are added to the target, or are discarded.

From Tables 6 and 7, we can see that:

(1) For GA-FS, about one third of genes are removed and two thirds are used for classification. Furthermore, the ratio of the number of input features to the number of output features for GA-MTL is similar to the ratio of the number of input features to the number of discarded features for GA-FS.

(2) H-MTL and GA-MTL-IR both use the same prediction risk criterion to discard irrelevant features, so the features discarded are the same and hence the number of discarded features are the same. The number of input features

Table 2: Mean and standard deviation (in parentheses) of correction scores (\%), calculated over 50 hold out runs.

\begin{tabular}{|c|c|c|c|c|c|c|}
\hline DATASET & ALL & GA-FS & H-MTL & GA-MTL & GA-MTL-IR & e-GA-MTL \\
\hline \multicolumn{7}{|c|}{$M=2$ for $\mathrm{ANN}$} \\
\hline Breast & $53.1(9.5)$ & $56.3(8.4)$ & $59.3(8.8)$ & $71.9(8.9)$ & $68.8(8.1)$ & $71.9(8.6)$ \\
\hline Colon & $57.1(7.8)$ & $61.9(7.3)$ & $66.6(7.1)$ & $80.9(6.3)$ & $85.7(6.4)$ & $80.9(6.4)$ \\
\hline Leukemia & $57.7(9.3)$ & $53.8(9.0)$ & $61.5(9.5)$ & $76.9(8.4)$ & $76.9(9.1)$ & $80.7(8.9)$ \\
\hline Ovarian & $57.1(6.4)$ & $67.9(7.4)$ & $75.0(5.4)$ & $78.6(5.9)$ & $79.8(5.1)$ & $82.1(5.8)$ \\
\hline Average & $56.3(8.3)$ & $60.0(8.0)$ & $65.6(7.7)$ & $77.1(7.4)$ & $77.8(7.2)$ & $78.9(7.4)$ \\
\hline \multicolumn{7}{|c|}{$M=10$ for $\mathrm{ANN}$} \\
\hline Breast & $64.3(8.5)$ & 67.3(8.7) & $65.7(8.3)$ & $75.4(8.3)$ & $71.8(7.5)$ & $75.8(8.0)$ \\
\hline Colon & $62.0(7.5)$ & $71.2(7.2)$ & $75.2(6.5)$ & $85.1(6.7)$ & $82.9(6.1)$ & $83.9(6.3)$ \\
\hline Leukemia & $65.3(8.8)$ & $69.2(8.4)$ & $76.3(8.8)$ & $84.6(8.9)$ & $83.1(8.1)$ & $85.6(8.3)$ \\
\hline Ovarian & $61.9(7.5)$ & $65.4(7.7)$ & $78.6(6.4)$ & $80.9(6.8)$ & $82.1(6.4)$ & $83.3(6.8)$ \\
\hline Average & $63.4(8.1)$ & $68.3(8.0)$ & $74.0(7.5)$ & $81.5(7.7)$ & $80.0(7.0)$ & $82.2(7.4)$ \\
\hline
\end{tabular}


Table 3: Mean and standard deviation (in parentheses) of sensitivity scores (\%), calculated over 50 hold out runs.

\begin{tabular}{|c|c|c|c|c|c|c|}
\hline DATASET & ALL & GA-FS & H-MTL & GA-MTL & GA-MTL-IR & e-GA-MTL \\
\hline \multicolumn{7}{|c|}{$M=2$ for $A N N$} \\
\hline Breast & $53.3(8.7)$ & $53.3(8.5)$ & $66.7(8.0)$ & $73.3(7.8)$ & $73.3(7.5)$ & $80.0(7.6)$ \\
\hline Colon & $28.6(7.4)$ & $28.6(7.5)$ & $42.8(7.4)$ & $71.4(7.0)$ & $85.7(7.1)$ & $71.4(7.1)$ \\
\hline Leukemia & $62.5(8.1)$ & $75.0(8.0)$ & $75.0(7.7)$ & 87.5(7.9) & $75.0(7.8)$ & $85.7(7.7)$ \\
\hline Ovarian & $60.0(7.5)$ & $73.3(7.4)$ & $76.7(7.4)$ & $80.0(6.7)$ & $80.0(6.9)$ & $83.3(7.2)$ \\
\hline Average & $5 I . I(7.9)$ & $57.6(7.9)$ & $65.3(7.6)$ & $78.1(7.4)$ & $78.5(7.3)$ & $80.1(7.4)$ \\
\hline \multicolumn{7}{|c|}{$M=10$ for $A N N$} \\
\hline Breast & $60.2(9.8)$ & $63.2(9.4)$ & $73.3(10.4)$ & $80.5(9.5)$ & $80.6(9.0)$ & $85.6(9.0)$ \\
\hline Colon & $42.7(7.8)$ & $43.4(7.5)$ & $51.2(7.4)$ & $72.1(7.5)$ & $72.3(7.1)$ & $72.3(6.8)$ \\
\hline Leukemia & $71.3(7.6)$ & $75.0(7.5)$ & $77.5(7.9)$ & $88.7(8.1)$ & $74.3(7.9)$ & $91.2(8.3)$ \\
\hline Ovarian & $64.7(7.5)$ & $71.4(7.3)$ & $80.5(7.4)$ & $81.2(7.0)$ & $80.0(6.9)$ & $86.7(7.1)$ \\
\hline Average & $59.7(8.2)$ & 63.3(7.9) & $70.6(8.3)$ & $80.6(8.0)$ & $76.8(7.7)$ & $84.0(7.8)$ \\
\hline
\end{tabular}

and output features are different, however. H-MTL has a predetermined number of input and output features; one quarter of the selected features are used for the input, and the other three quarters are added to the output. In contrast, for GA-MTL-IR, the features are determined by a genetic algorithm, but the ratio of the number of input features to the number of output features is similar to that of H-MTL.

(3) For e-GA-MTL, although the number of input, output and discarded features are determined automatically by the genetic algorithm, the ratios among these numbers are similar to those for H-MTL and GA-MTL-IR.

\section{Discussions}

We have demonstrated that genetic algorithm based multi-task learning (GA-MTL) methods perform better than the heuristic methods and feature selection methods, and that e-GA-MTL performs the best of all the methods considered. Several questions come immediately to mind:

Why does multi-task learning succeed?

In a previous study, Caruana et al. gave an explanation $[8,9]$ of why multi-task learning succeeds. Here we combine their results with the framework presented here. Yu and Liu [15] proposed to categorize the features into four classes, namely:

I: irrelevant features,

II: weakly relevant and redundant features,

III: weakly relevant but non-redundant features, and IV: strongly relevant features;

Table 4: Mean and standard deviation (in parentheses) of specificity scores (\%), calculated over 50 hold out runs.

\begin{tabular}{|c|c|c|c|c|c|c|}
\hline DATASET & ALL & GA-FS & H-MTL & GA-MTL & GA-MTL-IR & e-GA-MTL \\
\hline \multicolumn{7}{|c|}{$M=2$ for $A N N$} \\
\hline Breast & $53.0(9.9)$ & $58.8(8.6)$ & $52.9(9.0)$ & $70.7(8.7)$ & $64.7(9.2)$ & $64.8(9.2)$ \\
\hline Colon & $71.3(10.2)$ & $64.7(9.5)$ & 73.4(8.4) & $85.7(9.8)$ & $85.7(9.1)$ & $85.7(8.4)$ \\
\hline Leukemia & $55.6(7.5)$ & $44.4(8.3)$ & $55.4(8.1)$ & 7I.7(7.8) & $77.8(7.5)$ & $83.3(7.4)$ \\
\hline Ovarian & $55.6(6.1)$ & $64.9(6.2)$ & $74.4(6.1)$ & 77.7(7.6) & 79.7(7.3) & $81.5(7.0)$ \\
\hline Average & $58.5(8.4)$ & $58.6(8.1)$ & $64.3(8.0)$ & $76.1(8.4)$ & $76.3(8.2)$ & $78.8(8.0)$ \\
\hline \multicolumn{7}{|c|}{$M=10$ for $\mathrm{ANN}$} \\
\hline Breast & $48.1(8.8)$ & $55.4(8.4)$ & $65.1(8.0)$ & $69.2(8.1)$ & $63.9(9.6)$ & $66.8(8.4)$ \\
\hline Colon & $71.3(10.0)$ & $85.3(9.7)$ & $83.5(10.5)$ & $92.2(9.7)$ & $93.1(10.0)$ & $92.6(10.8)$ \\
\hline Leukemia & $65.4(8.5)$ & $77.4(7.8)$ & $75.6(8.1)$ & $64.8(6.8)$ & $78.4(6.2)$ & $91.5(6.0)$ \\
\hline Ovarian & $61.3(5.1)$ & $65.6(5.9)$ & $77.0(4.3)$ & $79.4(5.0)$ & $87.0(5.4)$ & $80.4(5.5)$ \\
\hline Average & $61.0(8.1)$ & $71.8(8.1)$ & $75.8(7.6)$ & $78.1(7.9)$ & $82.2(7.8)$ & $82.6(7.6)$ \\
\hline
\end{tabular}


Table 5: Mean and standard deviation (in parentheses) of precision scores (\%), calculated over 50 hold out runs.

\begin{tabular}{|c|c|c|c|c|c|c|}
\hline DATASET & ALL & GA-FS & H-MTL & GA-MTL & GA-MTL-IR & e-GA-MTL \\
\hline \multicolumn{7}{|c|}{$M=2$ for $A N N$} \\
\hline Breast & $50.0(8.8)$ & $53.3(8.4)$ & $55.5(8.4)$ & $68.8(8.1)$ & 64.7(7.9) & $66.7(8.2)$ \\
\hline Colon & $33.3(8.7)$ & $25.0(8.1)$ & $42.9(7.4)$ & $71.4(7.8)$ & $75.0(7.7)$ & $71.4(7.4)$ \\
\hline Leukemia & $38.5(7.7)$ & $37.5(7.9)$ & $42.8(7.5)$ & $58.3(7.6)$ & $60.0(7.4)$ & 66.7(7.1) \\
\hline Ovarian & $42.9(6.4)$ & $53.7(6.6)$ & $62.6(6.1)$ & $66.6(6.8)$ & $68.6(7.1)$ & $71.4(6.5)$ \\
\hline Average & $41.2(7.9)$ & $42.4(7.8)$ & $51.0(7.4)$ & $66.3(7.6)$ & $67.1(7.5)$ & $69.1(7.3)$ \\
\hline \multicolumn{7}{|c|}{$M=10$ for $A N N$} \\
\hline Breast & $42.6(9.2)$ & $60.0(9.0)$ & $65.4(9.7)$ & $82.4(8.4)$ & $82.1(9.2)$ & $82.6(9.1)$ \\
\hline Colon & $51.2(7.5)$ & $56.2(7.4)$ & 64.7(7.3) & $70.6(8.1)$ & $66.7(8.2)$ & 68.4(7.3) \\
\hline Leukemia & $46.1(7.8)$ & $63.1(7.4)$ & $63.6(7.6)$ & $57.0(7.4)$ & $60.0(6.4)$ & $67.1(6.8)$ \\
\hline Ovarian & $47.6(5.4)$ & $53.6(5.7)$ & $66.4(4.3)$ & $68.4(5.1)$ & $78.2(5.0)$ & $72.1(5.5)$ \\
\hline Average & $46.9(7.5)$ & $58.2(7.4)$ & $65.0(7.2)$ & $69.6(7.3)$ & $71.8(7.2)$ & $72.6(7.2)$ \\
\hline
\end{tabular}

where III and IV comprise the optimal feature subset and I and II should be removed using feature selection methods. We have found that II contains useful information. These features should not be discarded, but rather should be used in the learning process. Multi-task learning is a method to use these redundant features to improve the prediction accuracy of the base learning method, which accounts for its improved performance.

Why do genetic algorithms perform better than the heuristic method?

Our results demonstrate that genetic algorithm based multi-task learning methods outperform heuristic multitask learning methods. The chief reason why this is so is that the heuristic method considered here uses the feature ranking technique to select features for the input and the target, which does not consider feature redundancy and/ or feature interaction. At the same time, is somewhat arbi- trary to use a prespecified number of features for the input and the target. This is another factor which reduces the performance of the heuristic method. In contrast, when the genetic algorithm selects features for the input and the target, it simultaneously considers feature redundancy and/or feature interaction. So it automatically determines the number of features for the input and target. In fact, Kudo and Sklansky proved that genetic algorithms have a higher probability of finding better solutions to naive feature selection problems than other complete, heuristic and random algorithms [16]. Among the genetic algorithm based multi-task learning methods, e-GA-MTL performs better than GA-MTL-IR. The number of features removed by e-GA-MTL is determined automatically by the genetic algorithm, while the number removed by GAMTL-IR is prespecified. This is further evidence that genetic algorithm based approaches outperform heuristic approaches.

Table 6: Mean and standard deviation (in parentheses) of the number of features, calculated over 50 hold out runs, where the base learners are $A N N s$ with $M=2$ units in the hidden layer.

\begin{tabular}{|c|c|c|c|c|c|c|}
\hline & & Breast & Colon & Leukemia & Ovarian & Average \\
\hline GA-FS & input & $15564.4(2.1)$ & $897.3(4.3)$ & $3245.3(2.5)$ & $10037.4(3.4)$ & $7436.1(3.1)$ \\
\hline discarded & $8916.6(1.5)$ & $1103.6(3.2)$ & $3883.6(2.7)$ & $5116.6(3.8)$ & $4755.1(2.8)$ & \\
\hline H-MTL & input & $12547.4(6.5)$ & $1014.0(5.4)$ & $4007.8(2.5)$ & $8924.7(2.5)$ & $6623.5(4.2)$ \\
\hline output & $4 \mid 82.5(6.9)$ & $338.0(3.2)$ & $1335.9(3.4)$ & $2974.9(3.4)$ & $2207.8(4.2)$ & \\
\hline discarded & $775 I .1(5.3)$ & $648.2(4.3)$ & $1785.3(2.6)$ & $3254.6(2.5)$ & $3359.8(3.7)$ & \\
\hline GA-MTL & input & $15624.5(2.7)$ & $993.3(3.3)$ & $3324.7(2.0)$ & $10154.2(4.4)$ & $7524.2(3.1)$ \\
\hline output & $8856.5(2.9)$ & $1007.6(3.5)$ & $3804.3(3.7)$ & $4999.8(2.8)$ & $4667.1(3.2)$ & \\
\hline GA-MTL-IR & input & $12656.3(3.6)$ & $877.4(4.5)$ & $4231.6(2.9)$ & $7895.4(3.5)$ & $6415.2(3.6)$ \\
\hline output & $4073.6(4.3)$ & $474.4(4.6)$ & $1112.1(2.7)$ & $4004.0(3.1)$ & $2416.0(3.7)$ & \\
\hline discarded & $7751.1(5.3)$ & $648.2(4.3)$ & $1785.3(2.6)$ & $3254.6(2.5)$ & $3359.8(3.7)$ & \\
\hline e-GA-MTL & input & $12743.3(4.1)$ & $884.7(5.2)$ & $4296.4(2.9)$ & $10235.2(3.6)$ & $7954.4(4.0)$ \\
\hline output & $4097.4(4.3)$ & $486.4(4.4)$ & $1175.6(2.1)$ & $2354.4(4.5)$ & $2028.5(3.8)$ & \\
\hline discarded & $7765.2(5.4)$ & $660.0(5.1)$ & $1796.2(2.1)$ & $2449.2(3.6)$ & $3167.7(4.0)$ & \\
\hline
\end{tabular}


Table 7: Mean and standard deviation (in parentheses) of the number of features, calculated over 50 hold out runs, where the base learners are ANNs with $M=10$ units in the hidden layer.

\begin{tabular}{|c|c|c|c|c|c|c|}
\hline & & Breast & Colon & Leukemia & Ovarian & Average \\
\hline GA-FS & input & $15042.4(3.5)$ & $917.3(4.5)$ & $3456.3(2.5)$ & $9837.4(4.3)$ & $7313.4(3.7)$ \\
\hline discarded & $9438.6(1.5)$ & $1082.6(2.8)$ & $3672.6(2.9)$ & $5316.6(3.7)$ & $4877.6(2.7)$ & \\
\hline H-MTL & input & $12620.2(5.6)$ & $1042.3(4.5)$ & $4082.1(3.6)$ & $8847.3(2.1)$ & $6648.0(4.0)$ \\
\hline output & $4206.7(5.9)$ & $347.5(2.3)$ & $1360.7(3.1)$ & 2949.1(4.3) & $2216.0(3.9)$ & \\
\hline discarded & $7654.1(3.5)$ & $610.2(2.7)$ & $1686.3(5.2)$ & $3357.6(2.1)$ & $3327.1(3.4)$ & \\
\hline GA-MTL & input & $15153.3(2.5)$ & $1041.1(4.1)$ & $3435.4(4.3)$ & $10034.5(3.3)$ & $7416.1(3.6)$ \\
\hline output & $9327.7(2.7)$ & $959.0(2.5)$ & $3693.6(3.8)$ & $5119.5(3.4)$ & $4775.0(3.1)$ & \\
\hline GA-MTL-IR & input & $|254| .5(3.6)$ & $842.2(4.5)$ & $4325.6(2.8)$ & $7984.2(2.1)$ & $6423.4(3.3)$ \\
\hline output & $4285.4(4.3)$ & $547.6(4.6)$ & $1 \mid 17.1(2.0)$ & $3812.2(3.8)$ & $2440.6(3.7)$ & \\
\hline discarded & $7654.1(3.5)$ & $610.2(2.7)$ & $1686.3(5.2)$ & $3357.6(2.1)$ & $3327.1(3.4)$ & \\
\hline e-GA-MTL & input & $12700.3(4.1)$ & $854.7(4.1)$ & $4 \mid 47.1(2.9)$ & $10453.2(3.5)$ & $7038.8(3.7)$ \\
\hline output & $4154.4(4.5)$ & $486.4(4.4)$ & I272.7(2.7) & $2454.4(3.5)$ & $2092.0(3.8)$ & \\
\hline discarded & $7645.2(5.3)$ & $660.0(5.7)$ & $1846.2(2.4)$ & $2489.2(3.7)$ & $3160.2(4.3)$ & \\
\hline
\end{tabular}

What effect do irrelevant features have on multi-task learning?

The effect of multi-task learning on irrelevant features can be observed by comparing the results obtained by e-GAMTL, GA-MTL-IR, and GA-MTL; e-GA-MTL and GA-MTLIR remove irrelevant features, while GA-MTL does not. Here we observed that e-GA-MTL and GA-MTL-IR outperformed GA-MTL, especially for the sensitivity and BACC measures. This shows that irrelevant features will degrade the generalization performance of multi-task learning methods, and reduce the robustness of the methods; they should therefore be removed before the learning process.

\section{Conclusions}

Random search methods of multi-task learning (MTL), including GA-MTL (Genetic Algorithm based MTL), GAMTL-IR (GA-MTL with irrelevant features removed) and eGA-MTL (an enhanced version of GA-MTL) are shown to improve the accuracy of multi-task learning and to make multi-task learning more convenient to use. Experimental results on microarray data sets for tumor classification showed that genetic algorithm based multi-task learning performed better than H-MTL, a heuristic multi-task learning method, and GA-FS, a naive feature selection method based on genetic algorithms. Furthermore, our results showed that e-GA-MTL and GA-MTL-IR, which remove irrelevant features, performed better than GAMTL, which does not. e-GA-MTL, which employs a genetic algorithm with a two bit encoding to remove irrelevant features and select features for the input and output, performed best. Since analysis of microarray data sets is a high dimensional problem, our results demonstrate that multi-task learning techniques can be employed to improve prediction performance of tumor classification by using redundant genes. Furthermore, our results demonstrate that genetic algorithms can be employed to improve multi-task learning by discarding irrelevant features and by selecting the input and target features automatically; GA-MTL and e-GA-MTL are shown to to improve generalization performance of classifiers on microarray and other high-dimensional data sets.

\section{Methods}

Multi-task learning (MTL) $[8,9]$ is a form of inductive transfer. It is applicable to any learning method that can share some of what is learned between multiple tasks. The basic idea is to use the selected features as the input feature set and to combine the target values with some of the discarded features to form the target output.

\section{Previous studies}

There exist several heuristic search methods for multi-task learning [9,11]. Caruana and de Sa [9] used a filter feature selection model of the cross-entropy criterion and/or an embedded model of kernel regression to rank the features, then employed the top $n_{1}$ features as the input feature set, and added the top $n_{2}$ of the remaining features to the target, where $n_{1}$ and $n_{2}$ are predefined numbers. Li et al.[11] employed clustering algorithms to select the features, which are first clustered using Kohonen neural networks; the features near the center of clusters are then selected as the input feature subset, and when the other unselected features are ranked according to the Euclidean distance to the input, the first few features with the least distance to the input are selected to add to the target to form the output.

\section{H-MTL}

H-MTL (Heuristic Multi-Task Learning) is a heuristic method with embedded feature selection that is based on the work of Caruana and de Sa [9]. The embedded model employs the prediction risk criteria $[17,18]$, which evaluates features by computing the change in training accuracy when the features are replaced by their mean values:

$$
S_{i}=\mathrm{ATR}-\mathrm{ATR}\left(\overline{\mathrm{x}}^{\mathrm{i}}\right)
$$


where ATR is the training accuracy. $\operatorname{ATR}\left(\bar{x}^{i}\right)$ is the test accuracy on the training set defined by:

$$
\operatorname{ATR}\left(\overline{\mathbf{x}}^{i}\right)=\frac{1}{p} \sum_{j=1}^{p}\left(\tilde{y}\left(x_{j}^{1}, \ldots, \bar{x}^{i}, \ldots, x_{j}^{n}\right)=y_{j}\right),
$$

where $n$ is the number of features, $p$ is the number of instances, $\bar{x}^{i}$ is the mean value of the $i^{\text {th }}$ feature, and $\tilde{y}($ ) is the prediction value of the $j^{\text {th }}$ example with the $i^{\text {th }}$ feature replaced by its mean value. The features with zero value are removed, since these features are not useful for learning.

After the features with zero value are removed, the prediction risk criteria is used to rank the remaining features in ascending order; the top quarter of these features are added to the output, and the remaining three quarters are used as the input. The overall algorithm is summarized in Figure 3.

\section{GA-FS}

To show the effectiveness of multi-task learning methods, we also implemented a naive feature selection method named GA-FS (Genetic Algorithm based Feature Selection). In GA-FS, we use a binary chromosome with the same length as the feature vector, which equals 1 if the corresponding feature is selected for the input, and 0 if the feature is discarded. The fitness function is defined as

$$
\text { fitness }=\frac{1}{3} \mathrm{ATR}+\frac{2}{3} \mathrm{ATV}
$$

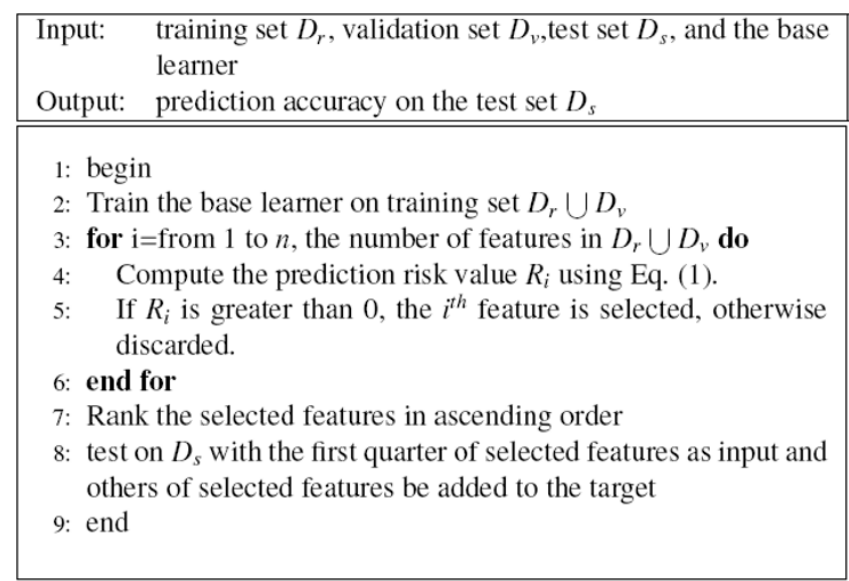

Figure 3

Heuristic multi-task learning (H-MTL) where ATR is the training accuracy of the base learning method, and ATV is the prediction accuracy on the validation data set.

The data set is divided into three parts: the training set $D_{r^{\prime}}$ the validation set $D_{v^{\prime}}$ and the test set $D_{s^{\prime}}$ as described in the Experimental Settings subsection below. The GA-FS approach is summarized in Figure 4.

\section{Genetic algorithm based multi-task learning}

In this subsection, we describe multi-task learning methods based on genetic algorithms. We previously proposed GA-MTL (Genetic Algorithm Based Multi-Task Learning), which did not consider irrelevant features. Here we propose two additional algorithms: GA-MTL-IR (GA-MTL with Irrelevant features Removed) and e-GA-MTL (an enhanced version of GA-MTL). GA-MTL-IR removes irrelevant features using an embedded feature selection method as in H-MTL, while e-GA-MTL removes irrelevant features using a genetic algorithm.

GA-MTL

In existing search methods $[9,11]$, the number of features selected for the input and/or the target is decided somewhat arbitrarily. In order to improve feature selection, GAMTL (Genetic Algorithm based Multi-Task Learning) $[10,14]$, a random method, employs a genetic algorithm [12] which simultaneously selects the features for both the input and the target. The number of features for the input and target is automatically determined by the method itself. In both GA-MTL and GA-FS, the same genetic algorithm is used for the feature selection task. The only difference between GA-MTL and GA-FS is the value of the binary chromosome; in GA-MTL, it equals 0 if the feature is selected to add to the output, whereas in GA-FS, it equals 0 if the feature is removed.

GA-MTL uses the fitness function defined by Equation (1). The data set is divided into three parts: the training set $D_{r^{\prime}}$

\begin{tabular}{|l}
\hline Input: $\begin{array}{l}\text { training set } D_{r}, \text { validation set } D_{v} \text {, test set } D_{s} \text {, and the base } \\
\text { learner } \\
\text { Output: prediction accuracy on the test set } D_{s}\end{array}$ \\
\hline \hline $\begin{array}{l}\text { 1: begin } \\
\text { 2: generate a population of weight vectors }\end{array}$ \\
3: evolve the population where the fitness of a weight vector $\mathbf{w}$ is \\
measured as in Eq. (2) on $D_{r}$ and $D_{v}$ \\
4: $\mathbf{w}^{*}=$ the evolved best weight vector \\
5: test on $D_{s}$ with features corresponding to 1 's in $\mathbf{w}^{*}$ as the input \\
and those to 0's be removed \\
6: end
\end{tabular}

Figure 4

Genetic algorithm based feature selection (GA-FS) 
the validation set $D_{y^{\prime}}$ and the test set $D_{s^{\prime}}$ as described in the Experimental Settings subsection below. The GA-MTL algorithm is summarized in Figure 5.

\section{GA-MTL-IR}

In GA-MTL, the irrelevant features are still present. These can be removed by many feature selection methods [6]. Here, we consider using the prediction risk criterion $[17,18]$ in an embedded method. As shown in Figure 6 , first the features with a prediction risk value of zero are removed, then GA-MTL is performed on the data set with the selected features. As this method removes the irrelevant features for GA-MTL, it is named GA-MTL-IR (GAMTL with Irrelevant features Removed).

\section{e-GA-MTL}

GA-MTL-IR removes irrelevant features using an embedded method, but it searches features for MTL using a genetic algorithm. Thus two search algorithms are used in GA-MTL-IR; why not instead use only a genetic algorithm? We propose an enhanced version of GA-MTL (e-GA-MTL), which is summarized in Fig. 7. It difiers from GA-MTL in its binary chromosome; instead of only one bit, two bits are used to represent each feature, where 00 means the corresponding feature is discarded, 10 means it is used as input, 01 means it is added to the output, and 11 means it is used as input and added to the output simultaneously.

\section{The base learning method}

Since artificial neural networks are a frequently used and powerful learning method, improved multi-layer perception neural networks were used as the base learning method. These are weight decay based neural networks in a Bayesian framework, which adds a regularization term to the objective function and are to some degree insensitive to the parameter settings [19].

\begin{tabular}{|l|}
\hline Input: $\quad \begin{array}{l}\text { training set } D_{r} \text {, validation set } D_{v} \text {, test set } D_{s} \text {, and the base } \\
\text { learner }\end{array}$ \\
Output: prediction accuracy on the test set $D_{s}$
\end{tabular}

\section{Figure 5}

Genetic algorithm based multi-task learning (GAMTL)

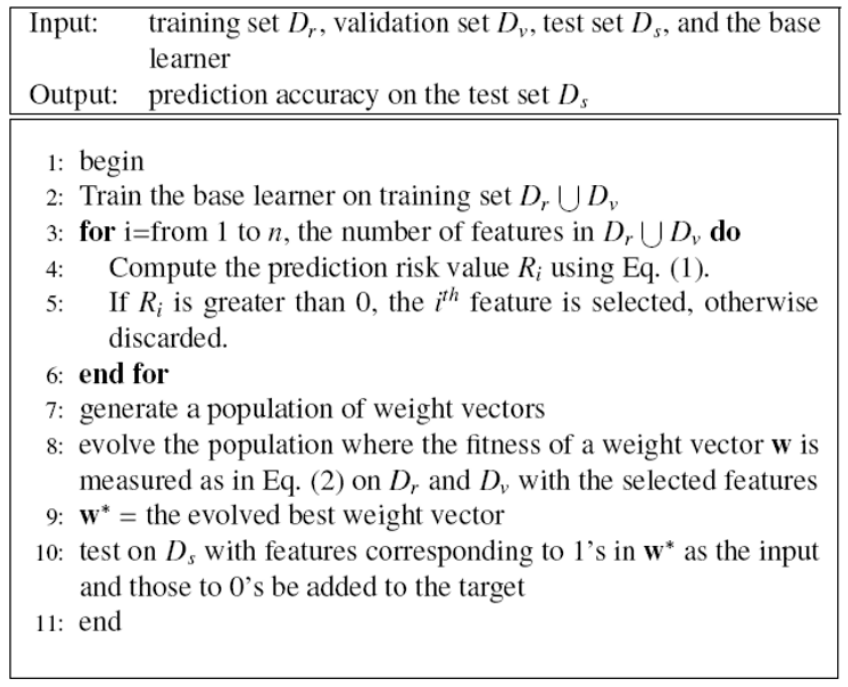

\section{Figure 6}

GA-MTL with irrelevant feature removed (GA-MTLIR)

\section{Experimental data sets}

The eight microarray data sets used in our study are listed in Table 8, and are briefy described below. Versions of the data files formatted for C4.5 are available [20].

Breast Cancer: Van't Veer et al.[21] used DNA microarray analysis on primary breast tumors and applied supervised classification methods to identify significant genes for the disease. The data contains 97 patient samples, 46 of which are from patients who had developed distance metastases within 5 years (labeled as "relapse"), the remaining 51 samples are from patients who remained free from the disease after their initial diagnosis for an interval of at least 5 years (labeled as "non-relapse"). The number of

\begin{tabular}{|l|}
\hline Input: $\quad \begin{array}{l}\text { training set } D_{r} \text {, validation set } D_{v} \text {, test set } D_{s} \text {, and the base } \\
\text { learner } \\
\text { Output: prediction accuracy on the test set } D_{s}\end{array}$ \\
\hline \hline 1: begin \\
2: generate a population of weight vectors \\
3: evolve the population where the fitness of a weight vector w is \\
measured as in Eq. (2) on $D_{r}$ and $D_{v}$ \\
4: $\mathbf{w}^{*}=$ the evolved best weight vector \\
5: test on $D_{s}$ with features corresponding to 10 's in $\mathbf{w}^{*}$ to be as \\
the input, those to 01 's be added to the target, those to 00 's be \\
discarded and those to 11 's be as the input and output simulta- \\
neously \\
6: end
\end{tabular}

Figure 7

Enhanced version of GA-MTL (e-GA-MTL) 
Table 8: Microarray data sets used for comparison

\begin{tabular}{llll}
\hline Data Sets & Samples & Class Ratio & Features \\
\hline Breast Cancer & 97 & $46 / 51$ & 24,481 \\
Colon & 62 & $22 / 40$ & 2,000 \\
Leukemia & 72 & $25 / 47$ & 7,129 \\
Ovarian & 253 & $91 / 162$ & 15,154 \\
\hline
\end{tabular}

genes is 24,481 and the missing values of " $\mathrm{NaN}^{\prime}$ are replaced with 100 .

Colon: Alon et al.[22] used Affymetrix oligonucleotide arrays to monitor expression levels of over 6,500 human genes from 40 tumor and 22 normal colon tissue samples. The 2,000 genes with the highest minimal intensity across the 62 tissues were used in the analysis.

Leukemia: The acute leukemia data set, published by Golub et al. [23], consists of 72 bone marrow samples with 47 ALL and 25 AML. The gene expression intensities are obtained from Affymetrix high-density oligonucleotide microarrays containing probes for 7,129 genes.

Ovarian: Petricoin et al. [24] identified proteomic patterns in serum to distinguish ovarian cancer from noncancer. The proteomic spectral data includes 91 controls (Normal) and 162 ovarian cancers; each sample contains the relative amplitude of the intensity at 15,154 molecular mass/charge $(\mathrm{M} / \mathrm{Z})$ identities.

\section{Experimental settings}

To evaluate the performance of the proposed approach, we use the hold out validation procedure. Each data set is used in its entirety, where split data sets are merged, and then the entire data set is randomly split into a training set and a testing set $D_{s} ; 2 / 3$ of the data is used for training and $1 / 3$ for testing. If a validation set is required, the training set is further split so that $2 / 3$ of the original training set is retained for training (forming the set $D_{r}$ ) and $1 / 3$ of the original training set is used for validation (forming the set $D_{v}$ ). classification results are reported for the test data sets $D_{s}$. This process is repeated 50 times.

The parameters of the genetic algorithms were set by default as in the MATLAB software, and we varied the parameters of the artificial neural networks to see how the settings of these parameters affected the results.

\section{Measures}

In order to precisely characterize the performance of different learning methods, we define several performance measures below (see [25]). Here TP, TN, FP, and FN, stand for the number of true positive, true negative, false positive, and false negative samples, respectively.
Sensitivity is defined as $\frac{T P}{T P+F N}$ and is also known as Recall.

Specificity is defined as $\frac{T N}{T N+F P}$.

BACC (Balanced Accuracy) is defined as $\frac{1}{2}\left(\frac{T P}{T P+F N}+\frac{T N}{T N+F P}\right)$, which defines the average of sensitivity and specificity.

Precision is defined as $\frac{T P}{T P+F P}$.

Correction is defined as $\frac{T P+T N}{T P+T N+F P+F N}$ and measures the overall percentage of samples correctly classified.

\section{Competing interests}

The authors declare that they have no competing interests.

\section{Authors' contributions}

Jack Y. Yang conceived and guided the project; Guo-Zheng $\mathrm{Li}$ proposed the idea; designed the algorithm and wrote the paper; Hao-Hua Meng performed the experiments; Youping Deng helped Guo-Zheng Li in writing the paper; Mary Qu Yang provided advice and help in designing the experiments.

\section{Acknowledgements}

This work was supported by the Nature Science Foundation of China under grant no. 20503015.

This article has been published as part of BMC Genomics Volume 9 Supplement I, 2008: The 2007 International Conference on Bioinformatics \& Computational Biology (BIOCOMP'07). The full contents of the supplement are available online at http://www.biomedcentral.com/|47|-2 |64/ 9? issue $=$ SI. .

\section{References}

I. Golub T, Slonim D, Tamayo P: Molecular classification of Cancer: Class Discovery and Class Prediction by Gene Expression. Bioinformatics \& Computational Biology 1999, 286:53 I-537.

2. Alon U: Broad Patterns of Gene Expression Revealed by Clustering Analysis of Tumor and Normal Colon Tissues Probed by Oligonucleotide Arrays. Proceedings of the National Academy of Sciences of the United States of America 1999:6745-6750.

3. Dudoit S, Fridlyand J, Speed T: Comparison of Discrimination Methods for the classification of Tumors Using Gene Expression Data. Journal of the American Statistical Association 2002, 457:77-87.

4. Guyon I, Weston J, Barnhill S, Vapnik V: Gene Selection for Cancer classification Using Support Vector Machines. Machine Learning 2002, 46:389-422.

5. Guyon I, Elisseeff A: An Introduction to Variable and Feature Selection. Journal of machine learning research 2003, 3: I I57-I I 82. 
6. Liu H, Yu L: Toward Integrating Feature Selection Algorithms for classification and Clustering. IEEE Transactions on Knowledge and Data Engineering 2005, I 7(3): I- I 2.

7. Yan J, Zhang B, Liu N, Yan S, Cheng Q, Fan W, Yang Q, Xi W, Chen Z: Effective and Efficient Dimensionality Reduction for Large-Scale and Streaming Data Preprocessing. Knowledge and Data Engineering, IEEE Transactions on 2006, 18:320-333.

8. Caruana R: Multitask Learning. Machine Learning 1997, 28:41-75.

9. Caruana R, de Sa VR: Benefiting from the variables that variable selection discards. Journal of machine learning research 2003, 3:1245-1264.

10. Yang JY, Li GZ, Liu LX, Yang MQ: classification of Brain Glioma by Using Neural Networks Ensemble with Multi-Task Learning. In Proceedings of The 2007 International Conference on Bioinformatics and Computational Biology (BIOCOMP'07) Las Vegas: CSREA Press; 2007:433-442.

II. Li GZ, Yang J, Lu J, Lu WC, Chen NY: On multivariate calibration problems. In ISNN 2004, Lecture Notes on Computer Science 3 I 73 Springer; 2004:389-394.

12. Goldberg DE: Genetic Algorithms in Search, Optimization, and Machine Learning. Boston: Addison Wesley; 1998.

13. Yang J, Honavar V: Feature Subset Selection Using a Genetic Algorithm. IEEE Intelligent Systems 1998, 13:44-49 [http://cite seer.ist.psu.edu/yang 98 feature.html].

14. Li GZ, Liu TY: Improving Generalization Ability of Neural Networks Ensemble with Multi-Task Learning. Journal of Computational Information Systems 2006, 2(4): I235-1239.

15. Yu L, Liu H: Eficient Feature Selection Via Analysis of Relevance and Redundancy. Journal of Machine Learning Research 2004, 5(Oct): I 205-I 224.

16. Kudo M, Sklansky J: Comparison of Algorithms that Select Features for Pattern classifiers. Pattern Recognition 2000, 33:25-4I.

17. Moody J, Utans J: Principled Architecture Selection for Neural Networks: Application to Corporate Bond Rating Prediction. In Advances in Neural Information Processing Systems Edited by: Edited by Moody JE, Hanson SJ, Lippmann RP. Morgan Kaufmann Publishers, Inc.; 1992:683-690.

18. Li GZ, Yang J, Liu GP, Xue L: Feature selection for multi-class problems using support vector machines. In Lecture Notes on artificial Intelligence 3/73 (PRICAI2004) Springer; 2004:292-300.

19. Foresee FD, Hagan MT: Gauss-Newton Approximation to Bayesian Regularization. In Proceedings of the 1997 International Joint Conference on Neural Networks 1997:1930-1935.

20. Li J, Liu H: Kent Ridge Bio-medical Data Set Repository. 2002 [http://sdmc.lit.org.sg/GEDatasets/Datasets.html].

21. Van't Veer LV, Dai H, Vijver MV, He Y, Hart A, Mao M, Peterse H, Kooy K, Marton M, Witteveen A, Schreiber G, Kerkhoven R, Roberts $\mathrm{C}$, Linsley P, Bernards R, Friend S: Gene expression profiling predicts clinical outcome of breast cancer. Nature 2002, 4I5(687I):530-536.

22. Alon U, Barkai N, Notterman DA, Gish K, Ybarra S, Mack D, Levine AJ: Broad patterns of gene expression revealed by clustering analysis of tumor and normal colon tissues probed by oligonucleotide arrays. Proceedings of the National Academy of Sciences of the United States of America 1999:6745-6750.

23. Golub TR, Slonim DK, Tamayo P, Huard C, Gaasenbeek M, Mesirov JP, Coller H, Loh ML, Downing JR, Caligiuri MA, Bloomfield CD, Lander ES: Molecular classification of Cancer: Class Discovery and Class Prediction by Gene Expression. Bioinformatics\&Computational Biology 1999, 286(5439):53I-537.

24. Petricoin EF, Ardekani AM, Hitt BA, Levine PJ, Fusaro VA, Steinberg SM, Mills GB, Simone C, Fishman D, Kohn EC, Liotta L: Use of proteomic patterns in serum to identify ovarian cancer. The Lancet 2002, 359(9306):572-577.

25. Levner I: Feature Selection and Nearest Centroid classification for Protein Mass Spectrometry. BMC Bioinformatics 2005 $6: 68$
Publish with Bio Med Central and every scientist can read your work free of charge

"BioMed Central will be the most significant development for disseminating the results of biomedical research in our lifetime. "

Sir Paul Nurse, Cancer Research UK

Your research papers will be:

- available free of charge to the entire biomedical community

- peer reviewed and published immediately upon acceptance

- cited in PubMed and archived on PubMed Central

- yours - you keep the copyright

Submit your manuscript here:

http://www.biomedcentral.com/info/publishing_adv.asp
BioMedcentral 